\title{
System Performance Analysis in IM/DD OFDM for Power Grid Utilizing Adaptive Volterra Equalization
}

\author{
Xia, Yong ${ }^{1}$; Cong, Hai Yang ${ }^{2}$; Zhu, Ming ${ }^{1}$; Ma, Wei Zhe ${ }^{1}$; Liu, Yang ${ }^{2}$; Qi, Zhi \\ Gang $^{3}$ \\ ${ }^{1}$ State Grid Benxi Electric Power Supply Company, Benxi 117000, China \\ ${ }^{2}$ State Grid Liaoning Electric Power Company Limited, Shenyang 110006, China \\ ${ }^{3}$ Liaoning Planning and Designing Institute of Post and Telecommunication Company Limited, \\ Shenyang 110011, China
}

\begin{abstract}
Keywords: Intensity-modulation and direct-detection; OFDM; Volterra filter; Power grid. Abstract. Intensity-modulation and direct-detection (IM/DD) orthogonal frequency division multiplexing (OFDM) systems have gained great attentions from researchers. However, the interaction between the chirp of direct modulation laser and fiber dispersion causes intermixing interferences among sub-carriers. Therefore, in this paper, we adopt Volterra filter to reduce the intermixing interferences and improve the BER performance in IM/DD OFDM systems. We first introduce the basic concept of Volterra filter and then set up simulation systems. The simulation results show that the system has a good BER performance by using a Volterra filter. This research provides a theoretical guidance for the information transmission in power grid.
\end{abstract}

\section{Introduction}

As a multi-carrier modulation technique, orthogonal frequency division multiplexing (OFDM) has been widely used in wireless local area networks, asymmetric digital subscriber line, and long-term evolution. In recent years, OFDM has also been applied to optical communication systems. The advantages of OFDM technology are as follows: 1) it has high spectrum efficiency due to the orthogonality of sub-carriers; 2) the OFDM signal can combat fiber dispersion since the symbol period is longer than that of a signal carrier; 3 ) digital signal processing can be utilized to compensate OFDM signal loss and distortion. Due to the advantages mentioned above, the optical OFDM has gained wide attentions from researchers all over the word [1-3].

According to different modulation and detection methods, optical OFDM systems can be divided into intensity modulation and direct detection (IM/DD), radio frequency up conversion and direct detection (RF/DD), radio frequency up conversion and coherent detection (RF/CO), and optical in-phase and quadrature modulation with $\mathrm{CO}$ detection (IQ/CO) [4]. Recently, IM/DD has been investigated for short-reach optical communication systems due to its low cost and operational complexity $[5,6]$. For IM/DD, in the transmitting end, a direct modulation laser (DML) such as a distributed feedback (DFB) laser, or a DFB with Mach-Zehnder modulator (MZM) can be utilized for intensity modulation, in order to generate optical OFDM signals; a photodiode is utilized for direct detection in the receiving end.

However, in IM/DD OFDM systems based on DML, the interaction between laser chirp and fiber dispersion will result in intermixing interferences among sub-carriers. Therefore, in this paper, we adopt a Volterra filter to reduce intermixing interferences and improve the BER performance in IM/DD OFDM systems. We establish simulation systems by using VPI simulator [7]. Simulation results show that the Volterra filter effectively reduces intermixing interferences among sub-carriers.

The rest of this paper is organized as follows. In section 2, we introduce tVolterra filter structure and working principles. Simulation setup is shown and simulation results are analyzed in Section 3. Finally, we conclude this paper in section 4. 


\section{Volterra filter structure and working principles}

Figure 1 shows the structure of the second-order Volterra filter. As in Fig. 1, the discrete input signal $X(n)=[x(n), x(n-1), \ldots, x(n-M+1)]$, the predicted output signal is $\hat{Y}(n)$. The second-order Volterra series expansion of this filter is as follows.

$$
\hat{Y}(n)=h_{0}+\sum_{m=0}^{M-1} h_{1}(m) \cdot x(n-m)+\sum_{m_{1}=0}^{M-1} \sum_{m_{2}=m_{1}}^{M-1} h_{2}\left(m_{1}, m_{2}\right) \cdot x\left(n-m_{1}\right) \cdot x\left(n-m_{2}\right),
$$

where, $M$ is the memory length, and it is equal to $2 . h_{0}$ is the constant term, and we let $h_{0}=0 . h_{1}(m)$ and $h_{2}\left(m_{1}, m_{2}\right)$ are the weighting coefficients of the linear and nonlinear components owned by the second-order Volterra filter, respectively. The filter coefficients can be adjusted by using least mean squares or recursive least squares algorithms.

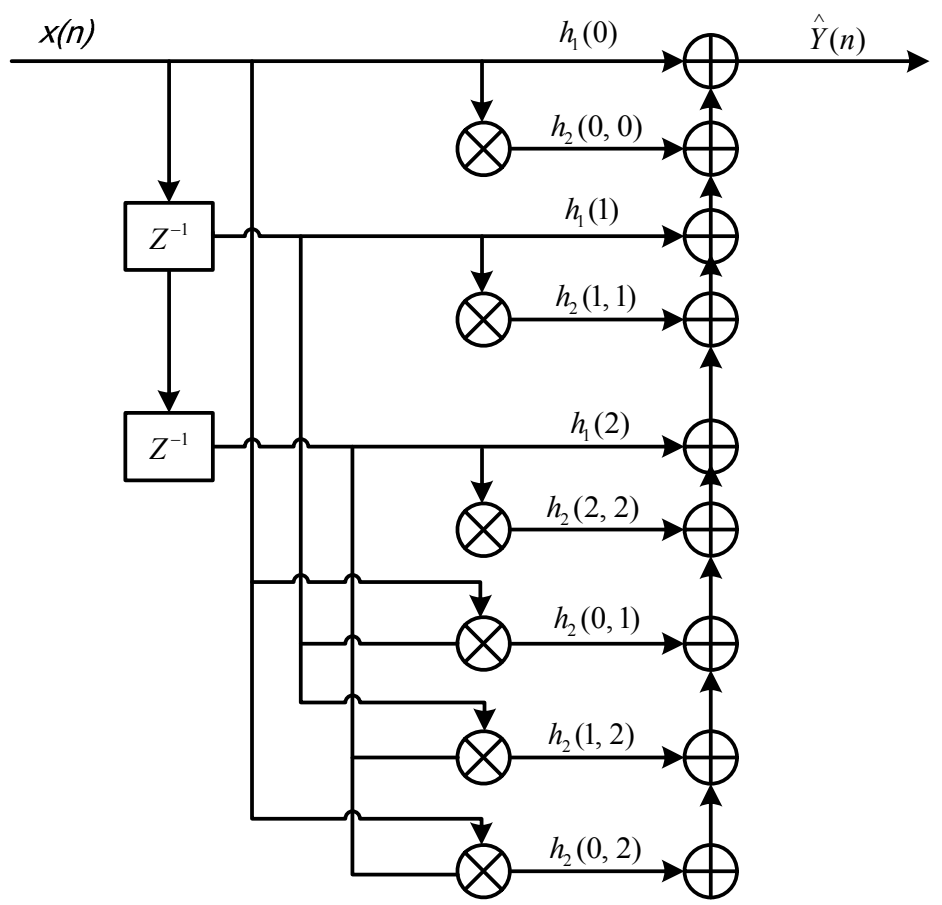

Fig. 1 Structure of second-order Volterra filter

In our IM/DD OFDM systems, the Volterra is utilized to equalize the received signal after analog to digital converter (DAC). We obtain the optimal coefficients via sending training symbols. By using the Volterra equalization, the nonlinear distortion also can be mitigated.

\section{Simulation setup and results}

The simulation setup is shown in Fig. 2, which is constructed by VPI TransmissionMaker 9.1. In the transmitting end, the OFDM coder module generates electrical OFDM signals. The basic OFDM parameter settings are as follows. The total number of sub-carriers is 64. Among which, 31 subcarriers carry payload; DC and Nyquist components are null; the data carried by the remaining 31 subcarriers are the complex conjugate of the sub-carriers carrying payload. As a result, a real-value data generates after Inverse Fast Fourier Transform (IFFT). The length of cycle prefix is 8 . We adopt $16-\mathrm{QAM}$ modulation. The baud rate is $18 \mathrm{Gbaud}$ and the net bit rate is $36 \mathrm{~Gb} / \mathrm{s}$. A raised cosine shaping stage with a roll-off factor $(0.2)$ is utilized to confirm the spectrum. The resulting signals pass through Digital to Analog Converter (DAC), and a laser driver that drives the DML, in sequence.

As for the laser driver, the direct current bias and peak-to-peak driving current are $30 \mathrm{~mA}$ and 15 $\mathrm{mA}$, respectively. 
In the receiving end, a photodiode is used for direct detection, in order to obtain electrical signals. Following is the Analog to Digital Converter (ADC) that generates discrete signals. Then, a Volterra filter is utilized to mitigate the nonlinear distortion. Finally, the signal is recovered by OFDM decoder module.

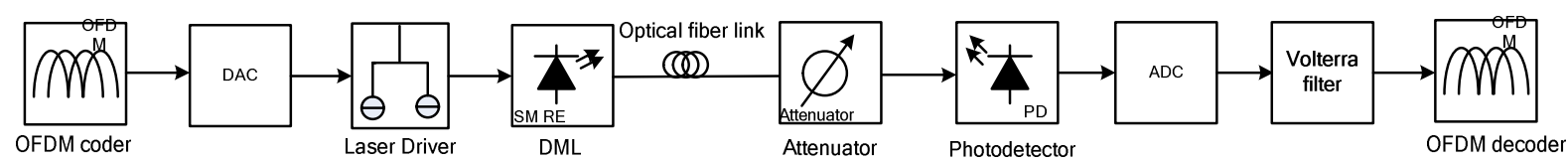

Fig. 2 Simulation setup

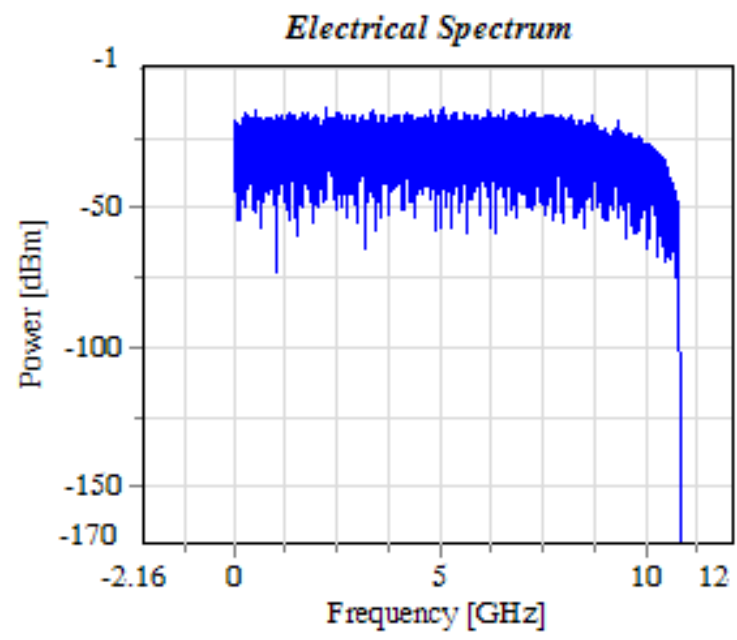

Fig. 3 Electrical spectrum in the transmitting end

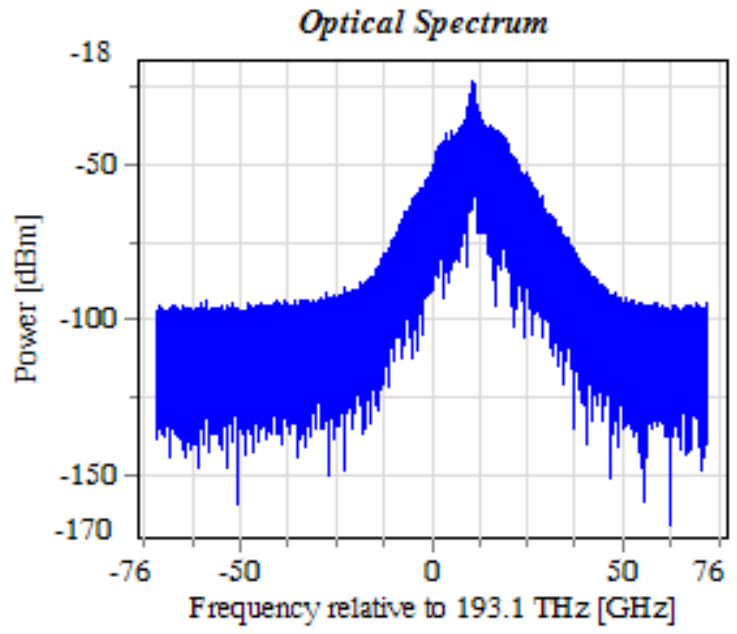

Fig. 4 Optical spectrum in the transmitting end

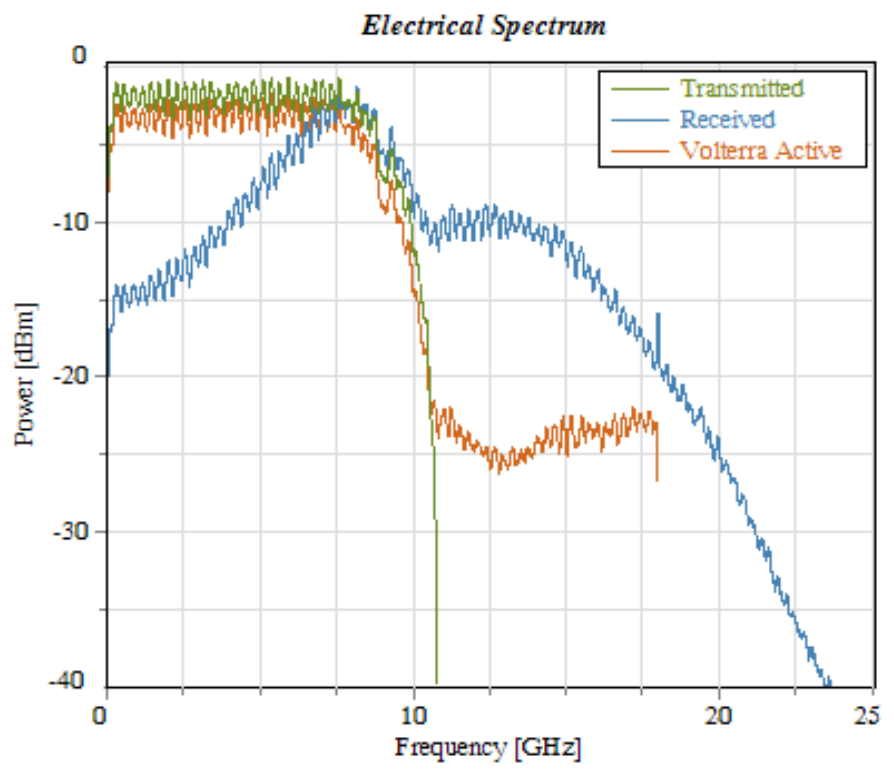

Fig. 5 Electrical spectrum in the receiving end

Figure 3 shows the electrical spectrum in the transmitting side. Since the roll-off factor is 0.2 , the bandwidth of the OFDM signal is $10.8 \mathrm{GHz}$. The optical spectrum in the transmitting side is depicted in Fig. 4. The central frequency offset of the optical carrier occurs due to the nonlinear frequency chirp of the DML. After $40 \mathrm{~km}$ fiber transmission, the received electrical spectrum with and without Volterra equalization are shown in Fig. 5. We can see that the electrical spectrum without Volterra equalization has a serious distortion due to the interaction between laser chirp and fiber dispersion. In particular, compared with the high frequency component, the low frequency component is attenuated more severely. By using the Volterra equalization, the transmitted signal can be well recovered. 
Figure 6 analyzes the BER variation for each sub-carrier. As expected, the system with the Volterra equalization has a better BER performance.

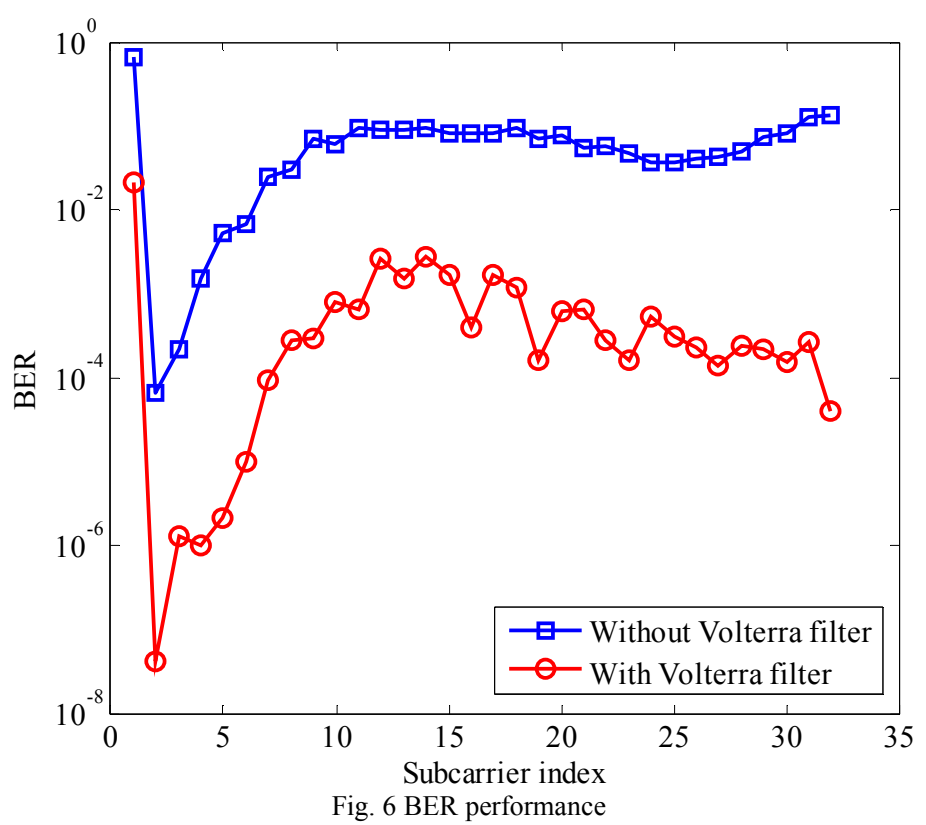

\section{Conclusion}

In this paper, we first introduced the Volterra filter structure and working principles, and then, our IM/DD OFDM system based on Volterra filter was set up by using VPI simulator. Simulation results showed that the Volterra filter effectively reduced the intermixing interferences among sub-carriers, and the BER performance was also improved. This study has some theoretical significance on the $\mathrm{IM} / \mathrm{DD}$ system design for power-grid communications.

\section{References}

[1] J. Armstrong. OFDM for optical communications, IEEE/OSA Journal of Lightwave Technology, 2009, 27(3), 189-204.

[2] N. André, H. Louchet, K. Habel, A. Richter. 33\% Capacity Improvement of a Direct-modulation Direct-detection OFDM Link Using Adaptive Volterra Equalization, Proc. ECOC, 1-3, 2014.

[3] N. Andre, K. Habel, H. Louchet, A. Richter. Adaptive nonlinear Volterra equalizer for mitigation of chirp-induced distortions in cost effective IMDD OFDM systems, Optics Express, 2013, 21(22): 26527-26532.

[4] E. Giacoumidis, A. Kavatzikidis1, N. P. Diamantopoulos, S. Mikroulis, I. Tomkos. Quantitative Comparison of Low-Cost OFDMA-PON Transceiver Implementations, Pro. ICTON, 1-4, 2012.

[5] L. Chen, B. Krongold, J. Evans. Theoretical characterization of nonlinear clipping effects in IM/DD optical OFDM systems, IEEE Transactions on Communications, 60(8), 2304-2312, 2012.

[6] C. Sanchez, B. Ortegea, J. L. Wei, J. M. Tang, J. Capmany. Analytical formulation of directly modulated OOFDM signals transmitted over an IM/DD dispersive link, Optics Express, 21(6), 7651-7666, 2013.

[7] http://www.vpiphotonics.com/ 